\title{
A Sharpened Nuclearity Condition for Massless Fields
}

\author{
W. DYBALSKI \\ Institut für Theoretische Physik, Universität Göttingen, Friedrich-Hund-Platz 1, \\ 37077 Göttingen, Germany. e-mail:dybalski@theorie.physik.uni-goettingen.de
}

Received: 10 March 2008 / Accepted: 18 May 2008

Published online: 5 June 2008

\begin{abstract}
A recently proposed phase space condition which comprises information about the vacuum structure and timelike asymptotic behavior of physical states is verified in massless free field theory. There follow interesting conclusions about the momentum transfer of local operators in this model.
\end{abstract}

Mathematics Subject Classification (2000). 81T05, 47B10, 46L40.

Keywords. quantum field theory, phase space analysis, automorphism groups.

\section{Introduction}

Compactness and nuclearity conditions, which characterize phase space properties, proved useful in the study of many aspects of Quantum Field Theory [4,5,7,10,14, $17,19]$. Verification of phase space conditions in models $[4,8,9,11,14,15]$ is an integral part of these investigations, since it demonstrates consistency of these criteria with the basic postulates of local, relativistic quantum physics [16]. In [15] a sharpened nuclearity condition has been proposed. It restricts correlations between different phase space regions and implies several physically desirable features. Among them are a certain form of additivity of energy over isolated subsystems and the uniqueness of vacuum states which can be prepared with a finite amount of energy. These vacuum states appear, in particular, as limits of physical states under large timelike translations in Lorentz covariant theories and are approximated by states of increasingly sharp energy-momentum values, in accordance with the uncertainty principle. This novel nuclearity condition seems also relevant to the study of particle aspects of a theory [12]. It is the aim of the present Letter to verify this criterion in massless free field theory. In comparison with the massive case studied in [15], the present investigation requires substantial technical improvements which we discuss below. As will be shown in a future publication, these advances enable a detailed harmonic analysis of translation automorphisms in massless theories.

Before we formulate the sharpened nuclearity condition, we recall briefly the mathematical framework: Let $V, W$ be Banach spaces and $\|\cdot\| \|$ be a norm on the space $\mathcal{L}(V, W)$ of linear maps from $V$ to $W$. We say that a map $\Pi: V \rightarrow W$ is 
$p$-nuclear w.r.t. the norm $\|\cdot\|$ if there exists a decomposition $\Pi(v)=\sum_{n} \Pi_{n}(v)$ into rank-one maps, convergent for any $v \in V$ in the norm topology in $W$, s.t. $v:=\left(\sum_{n}\left\|\Pi_{n}\right\|^{p}\right)^{\frac{1}{p}}<\infty$. The $p$-norm $\|\Pi\|_{p}$ of this map is the smallest such $v$ over the set of all admissible decompositions. To construct the norms which are suitable for our purposes, suppose that there acts a group of automorphisms $\mathbb{R}^{s+1} \ni x \rightarrow \beta_{x}$ on $V$. Then, for any $N \in \mathbb{N}$ and $x_{1} \ldots x_{N} \in \mathbb{R}^{s+1}$, we set

$$
\|\Pi\|_{x_{1} \ldots x_{N}}=\sup _{v \in V_{1}}\left(\sum_{k=1}^{N}\left\|\Pi\left(\beta_{x_{k}} v\right)\right\|^{2}\right)^{\frac{1}{2}}, \quad \Pi \in \mathcal{L}(V, W),
$$

where $V_{1}$ is the unit ball in $V$, and denote the corresponding $p$-norm by $\|\cdot\|_{p, x_{1} \ldots x_{N}}$.

Next, we identify the spaces $V, W$, automorphisms $\beta_{x}$ and maps $\Pi$ in the framework of Quantum Field Theory. Let $\mathcal{H}$ be the Hilbert space, $\omega_{0}$ the normal vacuum state, $\mathbb{R}^{s+1} \ni x \rightarrow \alpha_{x} \in \operatorname{Aut}(B(\mathcal{H}))$ the translation automorphisms and $H$ the Hamiltonian. We set $\mathcal{T}_{E}=P_{E} B(\mathcal{H})_{*} P_{E}$, where $P_{E}$ is the spectral projection of $H$ on the subspace spanned by vectors of energy lower than $E$ and choose $V=\check{\mathcal{T}}_{E}:=$ $\left\{\varphi-\varphi(I) \omega_{0} \mid \varphi \in \mathcal{T}_{E}\right\}$. This space is clearly invariant under the dual action of translations $\beta_{x}=\alpha_{x}^{*}$. Finally, we set $W=\mathfrak{A}(\mathcal{O})^{*}$, where $\mathfrak{A}(\mathcal{O}) \subset B(\mathcal{H})$ is the local algebra of observables attached to a double cone $\mathcal{O} \subset \mathbb{R}^{s+1}$, and define the family of maps $\Pi_{E}: \stackrel{\circ}{\mathcal{T}}_{E} \rightarrow \mathfrak{A}(\mathcal{O})^{*}$ given by

$$
\Pi_{E}(\varphi)=\left.\varphi\right|_{\mathfrak{A}(\mathcal{O})}, \quad \varphi \in \stackrel{\circ}{\mathcal{T}}_{E} .
$$

The strengthened nuclearity condition, proposed in [15], has the following form.

Condition $N_{\sharp}$. The maps $\Pi_{E}$ are $p$-nuclear w.r.t. the norms $\|\cdot\|_{x_{1} \ldots x_{N}}$ for any $N \in$ $\mathbb{N}, x_{1} \ldots x_{N} \in \mathbb{R}^{s+1}, 0<p \leq 1, E \geq 0$, and double cone $\mathcal{O} \subset \mathbb{R}^{s+1}$. Moreover, there holds for their nuclear $p$-norms

$$
\limsup \left\|\Pi_{E}\right\|_{p, x_{1} \ldots x_{N}} \leq c_{p},
$$

where $c_{p}$ is independent of $N$ and the limit is taken for configurations $x_{1} \ldots x_{N}$, where all $x_{i}-x_{j}, i \neq j$, tend to spacelike infinity.

We note that the first, qualitative part of this criterion is equivalent to Condition $N_{\sharp}$ formulated in [11] and the essential additional information is contained in the bound (3). This refinement is motivated by the observation that a measurement is always accompanied by an energy transfer from the physical state to the observable. Additivity of energy over isolated subregions should then imply that for any $\varphi \in \stackrel{\circ}{\mathcal{T}}_{E}$ the restricted functionals $\left.\alpha_{\vec{x}}^{*} \varphi\right|_{\mathfrak{A}(\mathcal{O})}$ are arbitrarily close to zero apart from translations varying in some compact subset of $\mathbb{R}^{s}$, depending on $\varphi$. This picture is particularly plausible in a massive theory, where a state of bounded energy contains only a finite number of particles which are well localized in space. Making use of this simplification, Condition $N_{\sharp}$ was verified in [15] in a theory of noninteracting massive particles. 
In the present Letter we demonstrate that this criterion is valid also in the massless case for $s \geq 3$. There the status of Condition $N_{\natural}$ is less obvious, since one has to handle the "infrared cloud" - states of bounded energy containing arbitrarily large numbers of massless particles whose localization properties are poor. The proof is accomplished by combining the underlying physical idea of additivity of energy over isolated subregions (Lemma 3.1) with the quadratic decay of vacuum correlations between spatially separated observables in a massless theory (Lemma 3.5). As an interesting application of our methods, we briefly discuss in the Conclusions the momentum transfer of local operators in the model under study.

\section{Massless Scalar Free Field Theory}

In the model at hand the Hilbert space $\mathcal{H}$ is the symmetric Fock space over $L^{2}\left(\mathbb{R}^{s}, \mathrm{~d}^{s} p\right)$. On this latter space there acts the unitary representation of translations

$$
\left(U_{1}(x) f\right)(\vec{p})=e^{i\left(\omega(\vec{p}) x^{0}-\vec{p} \vec{x}\right)} f(\vec{p}), \quad f \in L^{2}\left(\mathbb{R}^{s}, \mathrm{~d}^{s} p\right),
$$

where $\omega(\vec{p})=|\vec{p}|$. We denote by $U(x)$ its second quantization acting on $\mathcal{H}$, introduce the corresponding family of automorphisms of $B(\mathcal{H})$

$$
\alpha_{x}(\cdot)=U(x) \cdot U(x)^{*}
$$

and adopt the notation $A(x):=\alpha_{x}(A)$ for translated operators $A \in B(\mathcal{H})$. Next, we construct the local algebra $\mathfrak{A}(\mathcal{O})$ attached to the double cone $\mathcal{O}$, whose base is the $s$-dimensional ball $\mathcal{O}_{r}$ of radius $r$ centered at the origin in configuration space: We introduce the closed subspaces $\mathcal{L}^{ \pm}:=\left[\omega^{\mp \frac{1}{2}} \widetilde{D}\left(\mathcal{O}_{r}\right)\right]$, where tilde denotes the Fourier transform, represent the respective projections by the same symbol and consider the real linear subspace of $L^{2}\left(\mathbb{R}^{s}, \mathrm{~d}^{s} p\right)$

$$
\mathcal{L}=(1+J) \mathcal{L}^{+}+(1-J) \mathcal{L}^{-},
$$

where $J$ is the complex conjugation in configuration space. Then the local algebra is given by

$$
\mathfrak{A}(\mathcal{O})=\{W(f) \mid f \in \mathcal{L}\}^{\prime \prime},
$$

where $W(f)=e^{i\left(a^{*}(f)+a(f)\right)}$ and $a^{*}(f), a(f)$ are the creation and annihilation operators.

The rest of this section, which serves mostly to establish our notation, is devoted to the proof of the well-known fact $[3,11]$ that the maps $\Pi_{E}$ in this model are $p$-nuclear w.r.t. the standard norm on $\mathcal{L}\left(\stackrel{\circ}{\mathcal{T}}_{E}, \mathfrak{A}(\mathcal{O})^{*}\right)$. In the massive case the argument was outlined in [15], Appendix B, so it suffices here to give a brief sketch which stresses the modifications: First, our present construction of the trace-class 
operator $T$ differs from the choices made in the existing literature $[3,11,15]$ : Let $Q_{E}$ be the projection on states of energy lower than $E$ in the single-particle space, let $h \in D\left(\mathcal{O}_{r}\right)$ be real and s.t. $\widetilde{h}>0$. We choose $\frac{1}{2} \leq \gamma<\frac{s-1}{2}$ and define operators $T_{E, \pm}=\omega^{-\frac{1}{2}} Q_{E} \mathcal{L}^{ \pm}, T_{h, \pm}=\omega^{-\gamma} \widetilde{h}^{1 / 2} \mathcal{L}^{ \pm}$, where $\widetilde{h}$ is the corresponding multiplication operator in momentum space. By a slight modification of Lemma 3.5 from [11] one obtains that for $s \geq 3$ these operators satisfy $\left\|\left|T_{E, \pm}\right|^{p}\right\|_{1}<\infty,\left\|\left|T_{h, \pm}\right|^{p}\right\|_{1}<\infty$ for any $p>0$, where $\|\cdot\|_{1}$ denotes the trace norm. We define the operator $T$ as follows

$$
T=\left(\left|T_{E,+}\right|^{2}+\left|T_{E,-}\right|^{2}+\left|T_{h,+}\right|^{2}+\left|T_{h,-}\right|^{2}\right)^{\frac{1}{2}} .
$$

Making use of the fact [18] that for any $0<p \leq 1$ and any pair of positive operators $A, B$, s.t. $A^{p}, B^{p}$ are trace-class, there holds $\left\|(A+B)^{p}\right\|_{1} \leq\left\|A^{p}\right\|_{1}+\left\|B^{p}\right\|_{1}$, we get

$$
\left\|T^{p}\right\|_{1} \leq\left\|\left|T_{E,+}\right|^{p}\right\|_{1}+\left\|\left|T_{E,-}\right|^{p}\right\|_{1}+\left\|\left|T_{h,+}\right|^{p}\right\|_{1}+\left\|\left|T_{h,-}\right|^{p}\right\|_{1} \text { for } 0<p \leq 1 .
$$

Since $T$ commutes with $J$, it has a $J$-invariant orthonormal basis of eigenvectors $\left\{e_{j}\right\}_{1}^{\infty}$ and we denote the corresponding eigenvalues by $\left\{t_{j}\right\}_{1}^{\infty}$.

In order to construct an expansion of the map $\Pi_{E}$ into rank-one mappings, we evaluate a Weyl operator on some functional $\varphi \in \mathcal{T}_{E}$, rewrite it in a normal ordered form and expand it into a power series

$$
\begin{aligned}
\varphi(W(f))= & \mathrm{e}^{-\frac{1}{2}\|f\|^{2}} \sum_{m^{ \pm}, n^{ \pm} \in \mathbb{N}_{0}} \frac{i^{m^{+}+n^{+}+2 m^{-}}}{m^{+} ! m^{-} ! n^{+} ! n^{-} !} \times \\
& \times \varphi\left(a^{*}\left(f^{+}\right)^{m^{+}} a^{*}\left(f^{-}\right)^{m^{-}} a\left(f^{+}\right)^{n^{+}} a\left(f^{-}\right)^{n^{-}}\right),
\end{aligned}
$$

where $f=f^{+}+i f^{-}$and $f^{ \pm} \in \mathcal{L}^{ \pm}$are real in configuration space. Subsequently, we expand each function $f^{ \pm}$in the orthonormal basis $\left\{e_{j}\right\}_{1}^{\infty}$ of $J$-invariant eigenvectors of the operator $T: f^{ \pm}=\sum_{j=1}^{\infty} e_{j}\left\langle e_{j} \mid f^{ \pm}\right\rangle$. Then, making use of the multinomial formula, we obtain

$$
a^{(*)}\left(f^{ \pm}\right)^{m^{ \pm}}=\sum_{\mu^{ \pm},\left|\mu^{ \pm}\right|=m^{ \pm}} \frac{m^{ \pm} !}{\mu^{ \pm} !}\left\langle e \mid f^{ \pm}\right\rangle^{\mu^{ \pm}} a^{(*)}\left(\mathcal{L}^{ \pm} e\right)^{\mu^{ \pm}},
$$

where $\mu^{+}, \mu^{-}$are multiindices, and substitute these expansions to (10). In order to simplify the resulting expression, we define for any two pairs of multiindices $\bar{\mu}=$ $\left(\mu^{+}, \mu^{-}\right), \bar{v}=\left(v^{+}, v^{-}\right)$functionals $S_{\bar{\mu}, \bar{v}} \in \stackrel{\circ}{\mathcal{T}}_{E}^{*}$ given by

$$
S_{\bar{\mu}, \bar{\nu}}(\varphi)=\varphi\left(a^{*}(\mathcal{L} e)^{\bar{\mu}} a(\mathcal{L} e)^{\bar{\nu}}\right),
$$

where $a^{(*)}(\mathcal{L} e)^{\bar{\mu}}=a^{(*)}\left(\mathcal{L}^{+} e\right)^{\mu^{+}} a^{(*)}\left(\mathcal{L}^{-} e\right)^{\mu^{-}}$. Moreover, with the help of the formula

$$
\begin{aligned}
& \left(\Omega \mid\left[a\left(e_{1}\right),\left[\ldots,\left[a\left(e_{k}\right),\left[a^{*}\left(e_{k+1}\right),\left[\ldots,\left[a^{*}\left(e_{l}\right), W(f)\right], \ldots\right] \Omega\right)=\right.\right.\right.\right. \\
& \quad=e^{-\frac{1}{2}\|f\|^{2}} \prod_{n_{1}=1}^{k}\left\langle e_{n_{1}} \mid i f\right\rangle \prod_{n_{2}=k+1}^{l}\left\langle i f \mid e_{n_{2}}\right\rangle,
\end{aligned}
$$


one can express the factors $\left\langle e \mid f^{ \pm}\right\rangle^{\mu^{ \pm}}$, appearing in (11), in terms of normal functionals $\tau_{\bar{\mu}, \bar{v}} \in \mathfrak{A}(\mathcal{O})^{*}$ defined as in [15], Appendix B, (using methods from [3]). Then expression (10) takes the form

$$
\varphi(W(f))=\sum_{\bar{\mu}, \bar{v}} \tau_{\bar{\mu}, \bar{v}}(W(f)) S_{\bar{\mu}, \bar{v}}(\varphi) .
$$

In order to extend this formula to all $A \in \mathfrak{A}(\mathcal{O})$, we study its convergence properties: In the present case the norms of the functionals $\tau_{\bar{\mu}, \bar{v}}$ are not uniformly bounded in $\bar{\mu}, \bar{v}$. Instead, one obtains as in formula (B.7) of [15]

$$
\left\|\tau_{\bar{\mu}, \bar{\nu}}\right\| \leq \frac{4^{|\bar{\mu}|+|\bar{\nu}|}}{(\bar{\mu} ! \bar{\nu} !)^{\frac{1}{2}}}\left(\frac{(\bar{\mu}+\bar{\nu}) !}{\bar{\mu} ! \bar{\nu} !}\right)^{\frac{1}{2}} \leq \frac{2^{\frac{5}{2}(|\bar{\mu}|+|\bar{\nu}|)}}{(\bar{\mu} ! \bar{\nu} !)^{\frac{1}{2}}},
$$

where $|\bar{\mu}|=\left|\mu^{+}\right|+\left|\mu^{-}\right|$and $\bar{\mu} !=\mu^{+} ! \mu^{-}$!. Making use of the fact that for any $f_{1}, \ldots, f_{n} \in L^{2}\left(\mathbb{R}^{s}, \mathrm{~d}^{s} p\right)$ in the domain of $\omega^{\frac{1}{2}}$ there hold the so called energy bounds [11]

$$
\left\|a\left(\omega^{\frac{1}{2}} f_{1}\right) \ldots a\left(\omega^{\frac{1}{2}} f_{n}\right) P_{E}\right\| \leq(E)^{\frac{n}{2}}\left\|f_{1}\right\| \ldots\left\|f_{n}\right\|,
$$

we obtain the estimate

$$
\left\|S_{\bar{\mu}, \bar{\nu}}\right\| \leq E^{\frac{|\overline{\mid}|+|\bar{\nu}|}{2}}\left\|\omega^{-\frac{1}{2}} Q_{E} \mathcal{L} e\right\|^{\bar{\mu}}\left\|\omega^{-\frac{1}{2}} Q_{E} \mathcal{L} e\right\|^{\bar{\nu}} \leq E^{\frac{|\overline{\mid}|+|\bar{\nu}|}{2}} t^{\bar{\mu}} t^{\bar{\nu}} .
$$

With the help of the bounds (15) and (17) one verifies that for any $0<p \leq 1$

$$
\begin{aligned}
\sum_{\bar{\mu}, \bar{\nu}}\left\|\tau_{\bar{\mu}, \bar{v} \|^{p}}\right\| S_{\bar{\mu}, \bar{\nu}} \|^{p} \leq \sum_{\bar{\mu}, \bar{\nu}} \frac{\left(2^{5} E\right)^{\frac{1}{2} p(|\bar{\mu}|+|\bar{\nu}|)}}{(\bar{\mu} !)^{\frac{1}{2} p}(\bar{\nu} !)^{\frac{1}{2} p}} t^{p \bar{\mu}} t^{p \bar{\nu}} & =\left(\sum_{\mu^{+}} \frac{\left(2^{5} E\right)^{\frac{1}{2} p\left|\mu^{+}\right|}}{\left(\mu^{+} !\right)^{\frac{1}{2} p}} t^{p \mu^{+}}\right)^{4} \leq \\
& \leq\left(\sum_{k=0}^{\infty} \frac{\left(2^{5} E\right)^{\frac{1}{2} p k}\left\|T^{p}\right\|_{1}^{k}}{(k !)^{\frac{1}{2} p}}\right)^{4}
\end{aligned}
$$

where in the last step we set $k=\left|\mu^{+}\right|$and made use of the multinomial formula. This bound allows us to restate expression (14) as follows

$$
\Pi_{E}(\varphi)=\sum_{\bar{\mu}, \bar{v}} \tau_{\bar{\mu}, \bar{v}} S_{\bar{\mu}, \bar{v}}(\varphi), \quad \text { for } \varphi \in \stackrel{\circ}{\mathcal{T}}_{E},
$$

where the sum converges in the norm topology in $\mathfrak{A}(\mathcal{O})^{*}$ and there holds, in addition, $\left\|\Pi_{E}\right\|_{p} \leq\left(\sum_{\bar{\mu}, \bar{\nu}}\left\|\tau_{\bar{\mu}, \bar{\nu}}\right\|^{p}\left\|S_{\bar{\mu}, \bar{\nu}}\right\|^{p}\right)^{1 / p}<\infty$ for $0<p \leq 1$. This concludes the proof of the known fact that Condition $N_{\sharp}$ holds in massless free field theory $[3,11]$. In the next section we will use the same expansion (19) to verify Condition $N_{\natural}$. 


\section{Verification of Condition $\mathbf{N}_{\natural}$}

By definition of the nuclear $p$-norms and formula (19) there holds the bound

$$
\left\|\Pi_{E}\right\|_{p, x_{1} \ldots x_{N}} \leq\left(\sum_{\bar{\mu}, \bar{v}}\left\|\tau_{\bar{\mu}, \bar{v}}\right\|^{p}\left\|S_{\bar{\mu}, \bar{\nu}}\right\|_{x_{1} \ldots x_{N}}^{p}\right)^{\frac{1}{p}} .
$$

To verify Condition $N_{\natural}$ we have to find estimates on the norms $\left\|S_{\bar{\mu}, \bar{\nu}}\right\|_{x_{1} \ldots x_{N}}$ whose growth with $N$ can be controlled at large spacelike distances $x_{i}-x_{j}$ for $i \neq j$. The first step in this direction is taken in the following lemma which is inspired by Lemma 2.2 from [6]. In contrast to the bound from [15], Lemma 4.1, the present estimate is uniform in the particle number and depends only on the energy of the state in question. This result substantiates the underlying physical idea of additivity of energy over isolated subregions.

LEMMA 3.1. Suppose that $g \in L^{2}\left(\mathbb{R}^{s}, \mathrm{~d}^{s} p\right)$ and $\widetilde{h} g$ is in the domain of $\omega^{-\frac{1}{2}}$, where $\widetilde{h} \in \widetilde{D}\left(\mathcal{O}_{r}\right)$ appeared in the definition of the operator $T$ above. Then, for any $x_{1} \ldots x_{N} \in \mathbb{R}^{s+1}$, there holds the bound

$$
\begin{aligned}
\left\|P_{E} \sum_{k=1}^{N}\left(a^{*}(g) a(g)\right)\left(x_{k}\right) P_{E}\right\| \leq & \left.E \sup _{|\vec{p}| \leq E} \widetilde{h}(\vec{p})\right|^{-2}\left\{\left\|\omega^{-\frac{1}{2}} \widetilde{h} g\right\|^{2}+\right. \\
& \left.+(N-1) \sup _{i \neq j}\left|\left\langle\omega^{-\frac{1}{2}} \widetilde{h} g \mid U\left(x_{i}-x_{j}\right) \omega^{-\frac{1}{2}} \widetilde{h} g\right\rangle\right|\right\} .
\end{aligned}
$$

Proof. We pick single-particle vectors $\Psi_{1}, g_{1} \in L^{2}\left(\mathbb{R}^{s}, \mathrm{~d}^{s} p\right)$ and define $Q=$ $\sum_{k=1}^{N}\left(a^{*}\left(g_{1}\right) a\left(g_{1}\right)\right)\left(x_{k}\right)$. Then there holds

$$
\begin{aligned}
\left(\Psi_{1} \mid Q Q \Psi_{1}\right) & \leq \sum_{l=1}^{N}\left(\Psi_{1} \mid\left(a^{*}\left(g_{1}\right) a\left(g_{1}\right)\right)\left(x_{l}\right) \Psi_{1}\right) \sum_{k=1}^{N}\left|\left\langle U\left(x_{k}\right) g_{1} \mid U\left(x_{l}\right) g_{1}\right\rangle\right| \leq \\
& \leq\left(\Psi_{1} \mid Q \Psi_{1}\right)\left\{\left\|g_{1}\right\|^{2}+(N-1) \sup _{i \neq j}\left|\left\langle U\left(x_{j}\right) g_{1} \mid U\left(x_{i}\right) g_{1}\right\rangle\right|\right\},
\end{aligned}
$$

where we made use of the fact that $a\left(U\left(x_{k}\right) g_{1}\right) a\left(U\left(x_{l}\right) g_{1}\right) \Psi_{1}=0$ and of the Cauchy-Schwarz inequality. Since $\left(\Psi_{1} \mid Q \Psi_{1}\right)^{2} \leq\left(\Psi_{1} \mid Q Q \Psi_{1}\right)\left\|\Psi_{1}\right\|^{2}$, we obtain

$$
\begin{aligned}
& \sum_{k=1}^{N}\left(\Psi_{1} \mid\left(a^{*}\left(g_{1}\right) a\left(g_{1}\right)\right)\left(x_{k}\right) \Psi_{1}\right) \leq \\
& \quad \leq\left\|\Psi_{1}\right\|^{2}\left\{\left\|g_{1}\right\|^{2}+(N-1) \sup _{i \neq j}\left|\left\langle U\left(x_{j}\right) g_{1} \mid U\left(x_{i}\right) g_{1}\right\rangle\right|\right\} .
\end{aligned}
$$

Next, let $n \geq 1$ and $\Psi_{n} \in P_{E} \mathcal{H}$ be an $n$-particle vector s.t. the corresponding symmetric wave-function $\Psi_{n}\left(\vec{p}_{1} \ldots \vec{p}_{n}\right)$ belongs to $S\left(\mathbb{R}^{s \times n}\right)$. We also introduce a single-particle wave-function associated with $\Psi_{n}$ given by $\Psi_{1}\left(\vec{p}_{1}\right)_{\vec{p}_{2}, \ldots, \vec{p}_{n}=}$ 
$\left|\vec{p}_{1}\right|^{\frac{1}{2}} \widetilde{h}\left(\vec{p}_{1}\right)^{-1} \Psi_{n}\left(\vec{p}_{1}, \ldots \vec{p}_{n}\right)$, where we treat $\vec{p}_{2}, \ldots, \vec{p}_{n}$ as parameters. With the help of (23) we get

$$
\begin{aligned}
& \sum_{k=1}^{N}\left(\Psi_{n} \mid\left(a^{*}(g) a(g)\right)\left(x_{k}\right) \Psi_{n}\right)= \\
&= n \int \mathrm{d}^{s} p_{2} \ldots \mathrm{d}^{s} p_{n} \sum_{k=1}^{N}\left(\Psi_{1, \vec{p}_{2}, \ldots, \vec{p}_{n}} \mid\left(a^{*}\left(\omega^{-\frac{1}{2}} \widetilde{h} g\right) a\left(\omega^{-\frac{1}{2}} \widetilde{h} g\right)\right)\left(x_{k}\right) \Psi_{1, \vec{p}_{2}, \ldots, \vec{p}_{n}}\right) \leq \\
& \leq n \int \mathrm{d}^{s} p_{1} \ldots \mathrm{d}^{s} p_{n}\left|\widetilde{h}\left(\vec{p}_{1}\right)\right|^{-2}\left|\vec{p}_{1}\right|\left|\Psi_{n}\left(p_{1}, \ldots p_{n}\right)\right|^{2} \times \\
& \quad \times\left\{\left\|\omega^{-\frac{1}{2}} \widetilde{h} g\right\|^{2}+(N-1) \sup _{i \neq j}\left|\left\langle\omega^{-\frac{1}{2}} \widetilde{h} g \mid U\left(x_{i}-x_{j}\right) \omega^{-\frac{1}{2}} \widetilde{h} g\right\rangle\right|\right\} .
\end{aligned}
$$

Finally, we note that

$$
\begin{aligned}
& n \int \mathrm{d}^{s} p_{1} \ldots \mathrm{d}^{s} p_{n}\left|\widetilde{h}\left(\vec{p}_{1}\right)\right|^{-2}\left|\vec{p}_{1}\right|\left|\Psi_{n}\left(\vec{p}_{1}, \ldots \vec{p}_{n}\right)\right|^{2} \leq \\
& \quad \leq \sup _{|\vec{p}| \leq E}|\widetilde{h}(\vec{p})|^{-2} \int \mathrm{d}^{s} p_{1} \ldots \mathrm{d}^{s} p_{n}\left(\left|\vec{p}_{1}\right|+\cdots+\left|\vec{p}_{n}\right|\right)\left|\Psi_{n}\left(\vec{p}_{1}, \ldots \vec{p}_{n}\right)\right|^{2} \leq \\
& \quad \leq \sup _{|\vec{p}| \leq E}|\widetilde{h}(\vec{p})|^{-2} E\left\|\Psi_{n}\right\|^{2},
\end{aligned}
$$

where we made use of the fact that the wave-function is symmetric. Since the operators $\left(a^{*}(g) a(g)\right)\left(x_{k}\right)$ conserve the particle number and vectors of the form $\Psi=$ $c \Omega+\sum_{n=1}^{\infty} \Psi_{n}$, where $\|\Psi\|^{2}=|c|^{2}+\sum_{n=1}^{\infty}\left\|\Psi_{n}\right\|^{2}<\infty$, are dense in $P_{E} \mathcal{H}$, we easily obtain the bound in the statement of the lemma.

Our next task is to control the expressions appearing on the right-hand side of estimate (21). Lemma 3.2 below, which holds in particular for $\widetilde{F}(\vec{p})=|\vec{p}|^{-2}$, will be crucial in this respect. We start with some definitions: for any $\rho>0$ and some fixed $\epsilon>0$ we choose a function $\chi\left(\mathcal{O}_{\rho}\right) \in C_{0}^{\infty}\left(\mathbb{R}^{s}\right)$ s.t. $\chi\left(\mathcal{O}_{\rho}\right)(\vec{x})=1$ for $\vec{x} \in \mathcal{O}_{\rho}$ and $\chi\left(\mathcal{O}_{\rho}\right)(\vec{x})=0$ for $\vec{x} \notin \mathcal{O}_{\rho+\epsilon}$. We denote the operator of multiplication by $\chi\left(\mathcal{O}_{\rho}\right)$ in configuration space by the same symbol.

LEMMA 3.2. Suppose that $F \in S^{\prime}\left(\mathbb{R}^{s}\right)$ coincides with a bounded, measurable function in the region $\left\{\vec{y} \in \mathbb{R}^{s}|| \vec{y} \mid \geq \rho\right\}$ and its Fourier transform $\widetilde{F}$ is a positive, measurable function s.t. $\widetilde{F}^{1 / 2} \in L^{2}\left(\mathbb{R}^{s}, \mathrm{~d}^{s} p\right)+L^{\infty}\left(\mathbb{R}^{s}, \mathrm{~d}^{s} p\right)$. Then $\widetilde{F}^{1 / 2} \chi\left(\mathcal{O}_{\rho}\right)$ is a bounded operator and there holds

$$
\left\|\chi\left(\mathcal{O}_{\rho}\right) \widetilde{F} \chi_{\vec{x}}\left(\mathcal{O}_{\rho}\right)\right\| \leq c_{s, \rho, \epsilon} \sup _{|\vec{z}| \leq 2 \rho+3 \epsilon}|F(\vec{z}-\vec{x})| \quad \text { for }|\vec{x}| \geq 3(\rho+\epsilon)
$$

where $\chi_{\vec{x}}\left(\mathcal{O}_{\rho}\right)(\vec{y})=\chi\left(\mathcal{O}_{\rho}\right)(\vec{y}-\vec{x})$, the constant $c_{s, \rho, \epsilon}$ is independent of $\vec{x}$ and we denote the operator of multiplication by $\widetilde{F}$ in momentum space by the same symbol. 
Proof. In order to prove the first statement we make a decomposition $\widetilde{F}^{1 / 2}=$ $\widetilde{F}_{2}^{1 / 2}+\widetilde{F}_{\infty}^{1 / 2}$, where $\widetilde{F}_{2}^{1 / 2} \in L^{2}\left(\mathbb{R}^{s}, \mathrm{~d}^{s} p\right), \widetilde{F}_{\infty}^{1 / 2} \in L^{\infty}\left(\mathbb{R}^{s}, \mathrm{~d}^{s} p\right)$. Since $\widetilde{F}_{\infty}^{1 / 2}$ is a bounded operator, it suffices to consider $\widetilde{F}_{2}^{1 / 2} \chi\left(\mathcal{O}_{\rho}\right)$. We pick $f_{1}, f_{2} \in S\left(\mathbb{R}^{s}\right)$ and estimate

$$
\begin{aligned}
\left|\left\langle f_{1} \mid \widetilde{F}_{2}^{1 / 2} \chi\left(\mathcal{O}_{\rho}\right) f_{2}\right\rangle\right| & =(2 \pi)^{-\frac{s}{2}}\left|\int \mathrm{d}^{s} p \mathrm{~d}^{s} q \bar{f}_{1}(\vec{p}) \widetilde{F}_{2}^{1 / 2}(\vec{p}) \tilde{\chi}\left(\mathcal{O}_{\rho}\right)(\vec{p}-\vec{q}) f_{2}(\vec{q})\right| \leq \\
& \leq c\left\|\bar{f}_{1} \widetilde{F}_{2}^{1 / 2}\right\|_{1}\left\|\widetilde{\chi}\left(\mathcal{O}_{\rho}\right)\right\|_{2}\left\|f_{2}\right\|_{2} \leq c\left\|f_{1}\right\|_{2}\left\|\widetilde{F}_{2}^{1 / 2}\right\|_{2}\left\|\widetilde{\chi}\left(\mathcal{O}_{\rho}\right)\right\|_{2}\left\|f_{2}\right\|_{2},
\end{aligned}
$$

where in the second step we made use of the Young inequality ${ }^{1}$ [22] and in the last estimate we applied Hölder's inequality.

Next, we verify relation (26). If $|\vec{x}| \geq 3(\rho+\epsilon)$, then $|\vec{y}+\vec{x}| \leq 2 \rho+3 \epsilon$ implies $|\vec{y}| \geq$ $\rho$ and the expression

$$
\widetilde{F}_{\vec{x}}(\vec{p}):=(2 \pi)^{-\frac{s}{2}} \int \mathrm{d}^{s} y e^{-i \vec{p} \vec{y}} F(\vec{y}) \chi_{-\vec{x}}\left(\mathcal{O}_{2(\rho+\epsilon)}\right)(\vec{y})
$$

defines a bounded, continuous function. The operator of multiplication by $\widetilde{F}_{\vec{x}}$ in momentum space, denoted by the same symbol, satisfies the equality

$$
\chi\left(\mathcal{O}_{\rho}\right) \widetilde{F}_{\vec{x}} \chi_{\vec{x}}\left(\mathcal{O}_{\rho}\right)=\chi\left(\mathcal{O}_{\rho}\right) \widetilde{F} \chi_{\vec{x}}\left(\mathcal{O}_{\rho}\right)
$$

which can be verified by computing the matrix elements of both bounded operators between vectors from $S\left(\mathbb{R}^{s}\right)$, proceeding to configuration space and noting that the distributions $F$ and $\chi_{-\vec{x}}\left(\mathcal{O}_{2(\rho+\epsilon)}\right) F$ coincide on the resulting set of smearing functions. Moreover, we obtain from (28)

$$
\begin{aligned}
\left|\widetilde{F}_{\vec{x}}(\vec{p})\right| & \leq(2 \pi)^{-\frac{s}{2}} \int \mathrm{d}^{s} y\left|\chi\left(\mathcal{O}_{2(\rho+\epsilon)}\right)(\vec{y})\right| \sup _{|\vec{z}| \leq 2 \rho+3 \epsilon}|F(\vec{z}-\vec{x})|= \\
& =c_{s, \rho, \epsilon} \sup _{|\vec{z}| \leq 2 \rho+3 \epsilon}|F(\vec{z}-\vec{x})|,
\end{aligned}
$$

what concludes the proof of the lemma.

After this preparation we set $g=\mathcal{L}^{ \pm} e$ in Lemma 3.1 and undertake the study of the functions

$$
\mathbb{R}^{s+1} \ni x \rightarrow\left\langle\omega^{-\frac{1}{2}} \widetilde{h} \mathcal{L}^{ \pm} e \mid U(x) \omega^{-\frac{1}{2}} \widetilde{h} \mathcal{L}^{ \pm} e\right\rangle
$$

appearing on the right-hand side of estimate (21). We recall from our discussion in Section 2 that $\omega^{-\frac{1}{2}} \widetilde{h}^{1 / 2} \mathcal{L}^{ \pm}$are trace-class operators, so $\widetilde{h} g$ are in the domain

${ }^{1}$ The Young inequality states that for any positive functions $f \in L^{r_{1}}\left(\mathbb{R}^{s}, \mathrm{~d}^{s} p\right), g \in L^{r_{2}}\left(\mathbb{R}^{s}, \mathrm{~d}^{s} p\right)$, $h \in L^{r_{3}}\left(\mathbb{R}^{s}, \mathrm{~d}^{s} p\right)$, where $1 \leq r_{1}, r_{2}, r_{3} \leq \infty$ s.t. $\frac{1}{r_{1}}+\frac{1}{r_{2}}+\frac{1}{r_{3}}=2$, there holds the bound

$$
\int \mathrm{d}^{s} p \mathrm{~d}^{s} q f(\vec{p}) g(\vec{p}-\vec{q}) h(\vec{q}) \leq c_{r_{1}, r_{2}, r_{3}}\|f\|_{r_{1}}\|g\|_{r_{2}}\|h\|_{r_{3}} .
$$


of $\omega^{-\frac{1}{2}}$ as required in Lemma 3.1. A link with Lemma 3.2 is provided by the following identities

$$
\mathcal{L}^{ \pm}=\omega^{\mp \frac{1}{2}} \chi\left(\mathcal{O}_{r}\right) \omega^{ \pm \frac{1}{2}} \mathcal{L}^{ \pm}
$$

where $r$ is the radius of the ball entering into the definition of the subspaces $\mathcal{L}^{ \pm}$. The following result covers the case of translations in space.

LEMMA 3.3. Assume that $s \geq 3$ and let $e$ be a normalized eigenvector of the operator $T$ corresponding to the eigenvalue $t$. Then there holds

(a) $\left\langle\omega^{-\frac{1}{2}} \widetilde{h} \mathcal{L}^{-} e \mid U(\vec{x}) \omega^{-\frac{1}{2}} \widetilde{h} \mathcal{L}^{-} e\right\rangle=0$ for $|\vec{x}|>4 r$,

(b) $\left|\left\langle\omega^{-\frac{1}{2}} \widetilde{h} \mathcal{L}^{ \pm} e \mid U(\vec{x}) \omega^{-\frac{1}{2}} \widetilde{h} \mathcal{L}^{ \pm} e\right\rangle\right| \leq \frac{c_{s, r} t^{2}}{(|\vec{x}|+1)^{s-2}}$,

where the constant $c_{s, r}$ is independent of $\vec{x}$ and $e$.

Proof. To prove part (a) we set again $\chi_{\vec{x}}\left(\mathcal{O}_{r}\right)(\vec{y})=\chi\left(\mathcal{O}_{r}\right)(\vec{y}-\vec{x})$ and note that

$$
\begin{aligned}
& \left\langle\omega^{-\frac{1}{2}} \widetilde{h} \mathcal{L}^{-} e \mid U(\vec{x}) \omega^{-\frac{1}{2}} \widetilde{h} \mathcal{L}^{-} e\right\rangle= \\
& \quad=\left\langle\omega^{-\frac{1}{2}} \widetilde{h} \mathcal{L}^{-} e \mid \chi\left(\mathcal{O}_{2 r}\right) \chi_{\vec{x}}\left(\mathcal{O}_{2 r}\right) U(\vec{x}) \omega^{-\frac{1}{2}} \widetilde{h} \mathcal{L}^{-} e\right\rangle=0,
\end{aligned}
$$

for $|\vec{x}|>4 r$, since $h \in D\left(\mathcal{O}_{r}\right)$ and hence $\omega^{-\frac{1}{2}} \widetilde{h} \mathcal{L}^{-} e \in\left[\widetilde{D}\left(\mathcal{O}_{2 r}\right)\right]$. Due to the uniform bound

$$
\left|\left\langle\omega^{-\frac{1}{2}} \widetilde{h} \mathcal{L}^{ \pm} e \mid U(\vec{x}) \omega^{-\frac{1}{2}} \widetilde{h} \mathcal{L}^{ \pm} e\right\rangle\right| \leq\left\|\omega^{\gamma-\frac{1}{2}} \widetilde{h}^{1 / 2}\right\|_{\infty}^{2}\left\langle e \mid T_{h, \pm}^{2} e\right\rangle \leq\left\|\omega^{2 \gamma-1} \widetilde{h}\right\|_{\infty} t^{2},
$$

which involves the parameter $\gamma \in\left[\frac{1}{2}, \frac{s-1}{2}\right.$ [ from the definition of the operator $T$, there also follows the $(-)$ part of $(b)$. To prove the $(+)$ part we estimate

$$
\begin{aligned}
\left|\left\langle\omega^{-\frac{1}{2}} \widetilde{h} \mathcal{L}^{+} e \mid U(\vec{x}) \omega^{-\frac{1}{2}} \widetilde{h} \mathcal{L}^{+} e\right\rangle\right| & =\left|\left\langle\widetilde{h} \omega^{\frac{1}{2}} \mathcal{L}^{+} e \mid \chi\left(\mathcal{O}_{2 r}\right) \omega^{-2} \chi_{\vec{x}}\left(\mathcal{O}_{2 r}\right) \widetilde{h} \omega^{\frac{1}{2}} U(\vec{x}) \mathcal{L}^{+} e\right\rangle\right| \leq \\
& \leq t^{2}\left\|\omega^{2 \gamma+1} \widetilde{h}\right\|_{\infty}\left\|\chi\left(\mathcal{O}_{2 r}\right) \omega^{-2} \chi_{\vec{x}}\left(\mathcal{O}_{2 r}\right)\right\| .
\end{aligned}
$$

Now we are in position to apply Lemma 3.2: We set $\widetilde{F}(\vec{p})=|\vec{p}|^{-2}$. Then

$$
\widetilde{F}(\vec{p})^{1 / 2}=|\vec{p}|^{-1} \theta(-|\vec{p}|+1)+|\vec{p}|^{-1} \theta(|\vec{p}|-1) \in L^{2}\left(\mathbb{R}^{s}, \mathrm{~d}^{s} p\right)+L^{\infty}\left(\mathbb{R}^{s}, \mathrm{~d}^{s} p\right)
$$

and $F(\vec{x})=c_{s}|\vec{x}|^{-(s-2)}$, where $c_{s}=2^{\frac{s}{2}-2} \Gamma\left(\frac{s}{2}-1\right)$. We obtain for $|\vec{x}| \geq 6 r+3 \epsilon$

$$
\left\|\chi\left(\mathcal{O}_{2 r}\right) \omega^{-2} \chi_{\vec{x}}\left(\mathcal{O}_{2 r}\right)\right\| \leq \frac{c_{s, r}}{(|\vec{x}|-4 r-3 \epsilon)^{s-2}} .
$$

Making use of the uniform bound (34), we get the estimate from the statement of the lemma for a suitable constant $c_{s, r}$.

In order to obtain estimates on functions (31) valid for arbitrary spacelike translations $x$ we recall, in a slightly generalized form, the following result from [8]. 
LEMMA 3.4. Let $\delta>0$. Then there exists some continuous function $f(\omega)$ which decreases almost exponentially, i.e. $\sup _{\omega}|f(\omega)| e^{|\omega|^{\kappa}}<\infty$ for any $0<\kappa<1$, and which has the property that for any pair of operators $A, B$ such that $\Omega$ belongs to their domains and to the domains of their adjoints, satisfying

$$
\left(\Omega \mid\left[A, e^{i t H} B e^{-i t H}\right] \Omega\right)=0 \quad \text { for }|t|<\delta,
$$

there holds the identity $(\Omega \mid A B \Omega)=(\Omega \mid A f(\delta H) B \Omega)+(\Omega \mid B f(\delta H) A \Omega)$.

With the help of the above lemma we prove the desired bounds.

LEMMA 3.5. Assume that $s \geq 3$. Let $e \in L^{2}\left(\mathbb{R}^{s}, \mathrm{~d}^{s} p\right)_{1}$ satisfy $T e=$ te and $J e=e$. Then, for any $\varepsilon>0$ and $x \in \mathbb{R}^{s+1}$ s.t. $|\vec{x}| \geq\left|x^{0}\right|$, there hold the estimates

$$
\left|\left\langle\widetilde{h} \omega^{-\frac{1}{2}} \mathcal{L}^{ \pm} e \mid U(x) \widetilde{h} \omega^{-\frac{1}{2}} \mathcal{L}^{ \pm} e\right\rangle\right| \leq \frac{c_{s, r, \varepsilon} t^{2}}{\left(|\vec{x}|-\left|x^{0}\right|+1\right)^{s-2-\varepsilon}},
$$

where the constant $c_{s, r, \varepsilon}$ is independent of $x$ and $e$.

Proof. First, we define the operators $\phi_{+}(e)=a^{*}\left(\widetilde{h} \mathcal{L}^{+} e\right)+a\left(\widetilde{h} \mathcal{L}^{+} e\right), \phi_{-}(e)=$ $a^{*}\left(i \widetilde{h} \mathcal{L}^{-} e\right)+a\left(i \widetilde{h} \mathcal{L}^{-} e\right)$ and their translates $\phi_{ \pm}(e)(x)=U(x) \phi_{ \pm}(e) U(x)^{-1}$. Since the projections $\mathcal{L}^{ \pm}$and the multiplication operators $\widetilde{h}$ commute with $J$ and $J e=e$, the operators $\phi_{ \pm}(e)$ are just canonical fields and momenta of the free field theory localized in the double cone of radius $2 r$ centered at zero. We assume without loss of generality that $x^{0}>0$, introduce functions $F^{ \pm}(\tau)=\left\langle\widetilde{h} \mathcal{L}^{ \pm} e \mid \omega^{-1} U\left(\vec{x}+\tau \hat{e}_{0}\right) \widetilde{h} \mathcal{L}^{ \pm} e\right\rangle$ for $0 \leq \tau \leq x^{0}$, where $\hat{e}_{0}$ is the unit vector in the time direction, and consider the derivative

$$
\left|\frac{\mathrm{d} F^{ \pm}(\tau)}{\mathrm{d} \tau}\right|=\left|\left(\Omega \mid \phi_{ \pm}(e) \phi_{ \pm}(e)\left(\vec{x}+\tau \hat{e}_{0}\right) \Omega\right)\right|
$$

We define $\delta_{\tau}=|\vec{x}|-\tau-4 r$ and assume that $\delta_{\tau}>0$ for $0 \leq \tau \leq x^{0}$, i.e. $|\vec{x}|-x^{0}>4 r$. Then, by locality, $\phi_{ \pm}(e)$ and $\phi_{ \pm}(e)\left(\vec{x}+\tau \hat{e}_{0}\right)$ satisfy the assumptions of Lemma 3.4 with $\delta=\delta_{\tau}$. Making use of this result, we obtain

$$
\begin{aligned}
\left|\frac{\mathrm{d} F^{ \pm}(\tau)}{\mathrm{d} \tau}\right|= & \mid\left\langle\omega^{-\gamma} \widetilde{h} \mathcal{L}^{ \pm} e \mid \omega^{2 \gamma} f\left(\delta_{\tau} \omega\right) U\left(\vec{x}+\tau \hat{e}_{0}\right) \omega^{-\gamma} \widetilde{h} \mathcal{L}^{ \pm} e\right\rangle+ \\
& +\left\langle\omega^{-\gamma} \widetilde{h} \mathcal{L}^{ \pm} e \mid \omega^{2 \gamma} f\left(\delta_{\tau} \omega\right) U\left(-\vec{x}-\tau \hat{e}_{0}\right) \omega^{-\gamma} \widetilde{h} \mathcal{L}^{ \pm} e\right\rangle \mid \leq \\
\leq & \frac{2}{\delta_{\tau}^{2 \gamma}} t^{2}\|\widetilde{h}\|_{\infty} \sup _{\omega \geq 0}\left|\omega^{2 \gamma} f(\omega)\right| .
\end{aligned}
$$


Next, we set $\gamma=\frac{s-1-\varepsilon}{2}$ for $0<\varepsilon<1$ and arrive at the following estimate

$$
\begin{aligned}
\left|\left\langle\omega^{-\frac{1}{2}} \widetilde{h} \mathcal{L}^{ \pm} e \mid U(x) \omega^{-\frac{1}{2}} \widetilde{h} \mathcal{L}^{ \pm} e\right\rangle\right|=\left|F^{ \pm}\left(x^{0}\right)\right| & \leq\left|F^{ \pm}(0)\right|+\int_{0}^{x^{0}} \mathrm{~d} \tau\left|\frac{d F^{ \pm}(\tau)}{d \tau}\right| \\
& \leq \frac{c_{s, r, \varepsilon} t^{2}}{\left(|\vec{x}|-x^{0}-4 r\right)^{s-2-\varepsilon}}
\end{aligned}
$$

where in the last step we applied Lemma 3.3 and estimate (41). Since the left-hand side of relation (42) satisfies a uniform bound analogous to (34), we obtain the estimate in the statement of the lemma.

Now we are ready to prove the required bounds on the norms of the functionals $S_{\bar{\mu}, \bar{v}}$.

PROPOSITION 3.6. Given a family of points $x_{1} \ldots x_{N} \in \mathbb{R}^{s+1}$ we define $\delta(\underline{x})=$ $\inf _{i \neq j}\left(\left|\vec{x}_{i}-\vec{x}_{j}\right|-\left|x_{i}^{0}-x_{j}^{0}\right|\right)$. For $s \geq 3, \delta(\underline{x}) \geq 0$ and any $\varepsilon>0$ the functionals $S_{\bar{\mu}, \bar{v}}$ satisfy the bound

$$
\left\|S_{\bar{\mu}, \bar{\nu}}\right\|_{x_{1} \ldots x_{N}}^{2} \leq 16 c_{s, r, \varepsilon} \sup _{|\vec{p}| \leq E}|\widetilde{h}(\vec{p})|^{-2} E^{|\bar{\mu}|+|\bar{v}|} t^{2(\bar{\mu}+\bar{\nu})}\left\{1+\frac{N-1}{(\delta(\underline{x})+1)^{s-2-\varepsilon}}\right\},
$$

where the constant $c_{s, r, \varepsilon}$ appeared in Lemma 3.5 .

Proof. Making use of the fact that $S_{0,0}=0$, we can assume without loss of generality that $\bar{v} \neq 0$ and decompose it into two pairs of multiindices $\bar{v}=\bar{v}_{a}+\bar{v}_{b}$ in such a way that $\left|\bar{v}_{b}\right|=1$. Proceeding as in the proof of Proposition 4.4 in [15] (formulas (4.12) and (4.13)) we obtain the bound

$$
\left\|S_{\bar{\mu}, \bar{\nu}}\right\|_{x_{1} \ldots x_{N}}^{2} \leq 16 E^{|\bar{\mu}|+\left|\bar{v}_{a}\right|} t^{2\left(\bar{\mu}+\bar{v}_{a}\right)}\left\|P_{E} \sum_{k=1}^{N}\left(a^{*}(\mathcal{L} e)^{\bar{v}_{b}} a(\mathcal{L} e)^{\bar{v}_{b}}\right)\left(x_{k}\right) P_{E}\right\| .
$$

From Lemmas 3.1 and 3.5 we get

$$
\begin{aligned}
& \left\|P_{E} \sum_{k=1}^{N}\left(a^{*}(\mathcal{L} e)^{\bar{v}_{b}} a(\mathcal{L} e)^{\bar{v}_{b}}\right)\left(x_{k}\right) P_{E}\right\| \leq E \sup _{|\vec{p}| \leq E}|\widetilde{h}(\vec{p})|^{-2}\left\{\left\|\widetilde{h} \omega^{-\frac{1}{2}}(\mathcal{L} e)^{\bar{v}_{b}}\right\|^{2}+\right. \\
& \left.\quad+(N-1) \sup _{i \neq j}\left|\left\langle\widetilde{h} \omega^{-\frac{1}{2}}(\mathcal{L} e)^{\bar{v}_{b}} \mid U\left(x_{i}-x_{j}\right) \widetilde{h} \omega^{-\frac{1}{2}}(\mathcal{L} e)^{\bar{v}_{b}}\right\rangle\right|\right\} \\
& \quad \leq c_{s, r, \varepsilon} \sup _{|\vec{p}| \leq E}|\widetilde{h}(\vec{p})|^{-2} E t^{2 \bar{v}_{b}}\left\{1+\frac{N-1}{(\delta(\underline{x})+1)^{s-2-\varepsilon}}\right\} .
\end{aligned}
$$

Substituting inequality (45) into formula (44), we obtain the estimate in the statement of the proposition. 
We note that the bound from Proposition 3.6 has a similar structure to estimate (17) for the ordinary norms of $S_{\bar{\mu}, \bar{v}}$. Therefore, making use of formulas (20) and (18), we obtain

$$
\begin{aligned}
& \left\|\Pi_{E}\right\|_{p, x_{1} \ldots x_{N}} \leq \\
& \quad \leq 4 c_{s, r, \varepsilon}^{1 / 2} \sup _{|\vec{p}| \leq E}|\widetilde{h}(\vec{p})|^{-1}\left(\sum_{k=0}^{\infty} \frac{\left(2^{5} E\right)^{\frac{1}{2} p k}\left\|T^{p}\right\|_{1}^{k}}{(k !)^{\frac{1}{2} p}}\right)^{\frac{4}{p}}\left\{1+\frac{N-1}{(\delta(\underline{x})+1)^{s-2-\varepsilon}}\right\}^{\frac{1}{2}} .
\end{aligned}
$$

It follows that $\lim \sup _{\delta(\underline{x}) \rightarrow \infty}\left\|\Pi_{E}\right\|_{p, x_{1} \ldots x_{N}}$ satisfies a bound which is independent of $N$. Consequently, we get

THEOREM 3.7. Condition $N_{\natural}$ holds in massless scalar free field theory in $s \geq 3$ dimensional space.

\section{Conclusions}

In this work we verified the sharpened nuclearity condition $N_{\natural}$ in massless free field theory in spacetime of physical or higher dimension. This criterion guarantees the uniqueness of the vacuum state in the energy-connected component of the state space, in agreement with physical observations [15]. Nevertheless, it turns out to be consistent with a degenerate vacuum structure: Recall that massless free field theory has a spontaneously broken gauge symmetry $\mathbb{R} \ni \lambda \rightarrow \beta_{\lambda}$, corresponding to a shift of the pointlike localized field by a constant, which is defined on Weyl operators by

$$
\beta_{\lambda}(W(f))=e^{i \lambda\left(\widetilde{\omega^{1 / 2} f}\right)(0)} W(f) .
$$

This group of transformations gives rise to a family of pure, regular vacuum states

$$
\omega_{0}^{(\lambda)}(W(f))=e^{i \lambda\left(\widetilde{\omega^{1 / 2} f}\right)(0)} \omega_{0}(W(f))
$$

whose energy-connected components are, in fact, disjoint subsets of the state space for $s \geq 3$ [13]. This is no longer true for $s=2$ in which case Condition $N_{\natural}$, as well as the weaker Condition $N_{\sharp}$, does not hold due to singular infrared properties of this theory [11].

The methods developed in the present Letter are relevant to harmonic analysis of local operators $A \in \mathfrak{A}(\mathcal{O})$. We recall that in any relativistic quantum field theory there holds the bound [6]

$$
\sup _{\varphi \in \mathcal{T}_{E, 1}} \int \mathrm{d}^{s} p|\vec{p}|^{s+1+\varepsilon}|\varphi(\widetilde{A}(\vec{p}))|^{2}<\infty,
$$

for any $\varepsilon>0$, where $\widetilde{A}(\vec{p})$ is the Fourier transform of $A(\vec{x})$. Since the mollifier $|\vec{p}|^{s+1+\varepsilon}$ suppresses the contributions to $\varphi(\widetilde{A}(\vec{p}))$ with small momentum transfer, which become relevant at asymptotic times [1,20,21], we are interested in the 
minimal power of $|\vec{p}|$ for which the bound (49) is still valid. Making use of an improved variant of Lemma 3.1, one can show that for $s \geq 3$ there holds in massless free field theory

$$
\sup _{\varphi \in \mathcal{T}_{E, 1}} \int \mathrm{d}^{s} p|\vec{p}|^{2}|\varphi(\widetilde{A}(\vec{p}))|^{2}<\infty .
$$

With the help of a suitable sequence of functionals $\varphi_{n} \in \mathcal{T}_{E, 1}$, involving arbitrarily large number of particles, it can be verified that the power of the mollifier $|\vec{p}|^{2}$ cannot be further reduced on the whole local algebra $\mathfrak{A}(\mathcal{O})$ in this model. However, making use of the more refined expansion of the map $\Pi_{E}$ into rank-one mappings, developed in [3], one can construct a subspace of finite co-dimension in $\mathfrak{A}(\mathcal{O})$ on which there holds the bound

$$
\sup _{\varphi \in \mathcal{T}_{E, 1}} \int \mathrm{d}^{s} p|\varphi(\widetilde{A}(\vec{p}))|^{2}<\infty,
$$

familiar from massive free field theory [15]. This subspace contains, in particular, the elements of the fixed-point subalgebra of $\lambda \rightarrow \beta_{\lambda}$ whose vacuum expectation values vanish. These results, whose detailed proofs will be presented elsewhere, demonstrate the utility of the phase space methods in the development of a more detailed harmonic analysis of automorphism groups [2].

\section{Acknowledgements}

I would like to thank Prof. D. Buchholz for his continuing advice and encouragement in the course of this work. Financial support from Deutsche Forschungsgemeinschaft is gratefully acknowledged.

Open Access This article is distributed under the terms of the Creative Commons Attribution Noncommercial License which permits any noncommercial use, distribution, and reproduction in any medium, provided the original author(s) and source are credited.

\section{References}

1. Araki, H., Haag, R.: Collision cross sections in terms of local observables. Commun. Math. Phys. 4, 77-91 (1967)

2. Arveson, W.: The harmonic analysis of automorphism groups. In: Operator algebras and applications. I. Proceedings of Symposia in Pure Mathematics, vol. 38, pp. 255307. American Mathematical Society, Providence (1982)

3. Bostelmann, H.: Lokale Algebren und Operatorprodukte am Punkt, Ph.D. Thesis. Universität Göttingen (2000). http://webdoc.sub.gwdg.de/diss/2000/bostelmann/

4. Bostelmann, H.: Phase space properties and the short distance structure in quantum field theory. J. Math. Phys. 46, 052301-052318 (2005)

5. Bostelmann, H.: Operator product expansions as a consequence of phase space properties. J. Math. Phys. 46, 082304-082317 (2005)

6. Buchholz, D.: Harmonic analysis of local operators. Commun. Math. Phys. 129, 631-641 (1990) 
7. Buchholz, D.: Phase space properties of local observables and structure of scaling limits. Ann. Inst. H. Poincaré 64, 433-459 (1996)

8. Buchholz, D., D'Antoni, C., Longo, R.: The universal structure of local algebras. Commun. Math. Phys. 111, 123-135 (1987)

9. Buchholz, D., Jacobi, P.: On the nuclearity condition for massless fields. Lett. Math. Phys. 13, 313-323 (1987)

10. Buchholz, D., Junglas, P.: On the existence of equilibrium states in local quantum field theory. Commun. Math. Phys. 121, 255-270 (1989)

11. Buchholz, D., Porrmann, M.: How small is the phase space in quantum field theory? Ann. Inst. H. Poincaré 52, 237-257 (1990)

12. Buchholz, D., Summers, S.J.: Scattering in relativistic quantum field theory: fundamental concepts and tools. In: Francoise, J.-P., Naber, G., Tsun, T.S. (eds.) Encyclopedia of Mathematical Physics, vol. 4, pp. 456-465. Elsevier, New York (2006). Preprint math$\mathrm{ph} / 0509047$

13. Buchholz, D., Wanzenberg, R.: The realm of the vacuum. Commun. Math. Phys. 143, 577-589 (1992)

14. Buchholz, D., Wichmann, E.H.: Causal independence and the energy-level density of states in local quantum field theory. Commun. Math. Phys. 106, 321-344 (1986)

15. Dybalski, W.: A sharpened nuclearity condition and the uniqueness of the vacuum in QFT. Commun. Math. Phys. Preprint arXiv:0706.4049v2 (to appear)

16. Haag, R.: Local Quantum Physics. Springer, Berlin (1992)

17. Haag, R., Swieca, J.A.: When does a quantum field theory describe particles? Commun. Math. Phys. 1, 308-320 (1965)

18. Kosaki, H.: On the continuity of the map $\phi \rightarrow|\phi|$ from the predual of a $W^{*}$-algebra. J. Funct. Anal. 59, 123-131 (1984)

19. Lechner, G.: Construction of quantum field theories with factorizing S-matrices. Commun. Math. Phys. 277, 821-860 (2008)

20. Porrmann, M.: Particle Weights and their Disintegration I. Commun. Math. Phys. 248, 269-304 (2004)

21. Porrmann, M.: Particle weights and their disintegration II. Commun. Math. Phys. 248, 305-333 (2004)

22. Reed, M., Simon, B.: Methods of Modern Mathematical Physics. Part II: Fourier Analysis, Self-Adjointness. Academic Press, New York (1975) 